\title{
Game over - Strategies for addiction
}

\author{
Lopes L., Henriques F., Alfafar M., Farinha R. Centro Hospitalar do Médio Tejo, Psiquiatria, Tomar, Portugal.
}

\section{INTRODUCTION}

Science has shown how gaming can benefit our daily lives. The video game industry had a revenue of over 138.7 billion dollars worldwide in 2018. There are 2.6 billion players of all ages (mean 30 years old) from all the world. Adolescents play more from Monday to Friday (up to 13 hours), adults play in a single gaming session and up to 27.76 hours per week. Many researchers working for the industry study big data on what players do in games, what keeps them playing and how do they spend money. Most are casual players, but some (0.5-6\%) will become addicted. Studies show that people with Internet Gaming Disorder (IGD), by DSM5, can have personality traits such as, impulsivity, and depression or attention deficit hyperactivity disorder tendencies.

We describe 3 popular games, Fortnite by Epic Games, League of Legends (LOL) by Riot Games, and Summoners War by Com2uS.

What is it that makes them so addictive?

Materials and methods: review of existing literature on IGD, as well as field research on popular online video games such as Fortnite, League of Legends and Summoners War.

\section{FORTNITE}

250 million accounts in March 2019. Free to play, multiplatform, player versus player game. Players kill each other, the winner is the last one standing. Can be played in teams. Players catch random items in odd places of the map (weapons, health, construction items for barricades). High risk players, will spawn in highly populated areas of the map in search for more action, while low risk players go to remoted areas. There is an experience bar and other bars from tasks hard to complete. When dying, the last image shown is the life bar of the winner, giving a sense of "near miss" and wanting to try again. Players always see their avatar and create identity through skins, accessories, dances and comedy. Some of these features are paid.

\section{LEAGUE OF LEGENDS}

100 million active users/month in 2016. Free to play, computer platform, multiplayer online battle arena. 2 teams of 5 try to defeat each other, destroying the other team's base. Players control one of 145 champions with different abilities. Matches take between 20-40 minutes and are never equal. Players' rewards include game currency and climbing a division rank seen by all. Real money is spent on skins with new animations for champions, but they give no tactical advantage. Riot Games changes the game every 2 weeks.

\section{SUMMONERS WAR}

100 million downloads. Free to play, on smartphone, massively multiplayer online role-playing game. Players collect pets to fight other players. They get experience points and rewards to empower their pets. Daily tasks and special events offer rewards, some functions are automated, and players check on them often. Players are compelled to spend money for the higher chance of getting better pets, seeing the best pets used by others and being redirected to the game store.
All these games have the following to create flow: sense of identity, autonomy, objectives and feeling of competence, social proof, social interaction, fear of missing out, positive reward, positive prediction error, a small uncertainty, investment (time and money), anchoring, luck, sense of discovery, near miss feelings and a loop to the store. They also add bright colors, animations and ring sounds to the awards. Still, the ones who get addicted are a minority.

\section{BRAIN FINDINGS IN IGD}

One study noted brain changes in-game of a player population.

LOL loading screen activates ventrolateral prefrontal cortex (VLPFC) and simultaneously deactivates dorsolateral prefrontal cortex (DLPFC) and frontal pole area (FPA).

VLPFC and DLPFC activity raises on $1^{\text {st }}$ enemy contact.

Game events (slay enemy, assist slaying enemy, slain by enemy) had different responses from DLPFC and FPA (attentional states shifts and cognitive resources), while VLPFC (cognitive demand for visuo-motor interactions) kept a similar activity but walking didn't produce any activation.

Studies in IGD watch players reactions to game screenshots. Amount of play correlates with cortical thickness of DLPFC. Increased volume of caudate and nucleus accumbens.

Game addiction is correlated with level of temporo-occipital and frontoparietal networks activation.

higher craving deactivated temporo-insular network.

Altered resting state activity in the orbitofrontal cortex, striatum, and sensory regions (impulse control, reward processing, and somatic representation of past experiences).

$>$ Increased activation of posterior cingulate cortex (reward at uncertainty and reflecting risk preferences in addictions).

\section{TREATMENT}

Studies on psychopharmacology show that bupropion may hold some promise on IGD treatment. Cognitive-behavioral strategies and motivational interventions may be useful, but evidence is still limited.

These may be intended to assist the patient to regain control over their gaming behavior instead of abstinence.

\section{CONCLUSION}

For better results in treatment of IGD we need to understand brain mechanisms in game learning and game addiction during play, understand why are some games more addictive than others and how to revert those changes in a total or partial way.

Weinstein, A., Livny, A. \& Weizman, A. New developments in brain research of internet and gaming disorder. Neurosci. Biobehav. Rev. 75, 314-330 (2017). Thalemann, R., Wo, K. \& Gru, S. M. Specific Cue Reactivity on Computer Game-Related Cues in Excessive Gamers. Behav. Neurosci. 121, 614-618 (2007). Zastrow, M. Is video game addiction really an addiction? Proc. Natl. Acad. Sci 114, 4268-4272 (2017). Dong, G., Wang, L., Du, X. \& Potenza, M. N. Gender-related differences in neural responses to gaming cues before and after gaming : implications for gender-specific vulnerabilities to Internet gaming disorder. Soc. Cogn. Affect. Neurosci. 1203-1214 (2018). Banz, B. C. et al. HHS Public Access. Prog. Brain Res. 1-16 (2019). Li, Y., Zhang, L., Long, K., Gong, H. \& Lei, H. Real-time monitoring prefrontal activities during online video game playing by functional near-infrared spectroscopy. $J$. Biophotonics James, R. J. E., Malley, C. O., Tunney, R. J. \& Hayden, B. Why are Some Games More Addictive than Others: The Effects of Timing and Payoff on Perseverance in a Slot Machine Game. Front. Psychol. 7, 1-11 (2016). Clarck, O. CGD Vault (2015). 